\title{
Anaphylaxis with angioedema by rocuronium during induction of general anesthesia -A case report-
}

\author{
Won Ju Jeong, Woon Young Kim, Joo Hyung Son, Yoon-Sook Lee, Jae Hwan Kim, and Young Cheol Park \\ Department of Anesthesiology and Pain Medicine, Korea University College of Medicine, Ansan Hospital, Ansan, Korea
}

Perioperative anaphylaxis is characterized by severe respiratory and cardiovascular manifestations. Correct management of anaphylaxis during anaesthesia requires a multidisciplinary approach with prompt recognition and treatment of the acute event by the attending anesthesiologist. A 34-year-old woman was scheduled to undergo endo venous laser therapy of varicose veins. She had no history of allergies and had never undergone general anesthesia. General anesthesia was induced with propofol and rocuronium bromide. Approximately three minutes after rocuronium administration, hypotension and tachycardia developed and angioedema around the eyelids and skin rashes and urticaria appeared. The patient received ephedrine and hydrocortisone with hydration. After achieving stable vital signs and symptom relief, surgery was performed without complications. A postoperative skin dermal test performed to identify the agent responsible revealed a positive skin test for rocuronium. (Korean J Anesthesiol 2010; 58: 391-395)

Key Words: Anaphylaxis, Angioedema, Intradermal skin test, Rocuronium.

General anesthesia involves the administration of a variety of medications to a patient in relatively short duration. Various side effects can occur due to reactions to the drugs provided with anaphylaxis being a life threatening severe reaction. Although anaphylaxis is a rare side effect, it can lead to death if not diagnosed treated quickly and appropriately. The clinical features of immediate hypersensitivity allergic reactions include not only hemodynamic changes but also urticaria, flare, angioedema, among which the observation of a skin reaction is useful for diagnosis and treatment. Moreover, identifying the causative agent is the most important procedure to prevent a recurrence [1,2].

We report an experience of anaphylaxis accompanied with angioedema after administrating propofol and rocuronium to induce general anesthesia in a 34-year-old female patient with varicose veins, who was scheduled to undergo endovenous laser therapy and varicosectomy, with a review of the relevant literature.

Received: June 15, 2009. Revised: 1st, June 23, 2009; 2nd, July 6, 2009. Accepted: July 7, 2009.

Corresponding author: Woon Young Kim, M.D., Department of Anesthesiology and Pain Medicine, Korea University College of Medicine, Ansan Hospital, 516, Gojan-dong, Ansan 425-707, Korea. Tel: 82-31-412-5297, Fax: 82-31-412-5294, E-mail: ckssis@korea.ac.kr

(c) This is an open-access article distributed under the terms of the Creative Commons Attribution Non-Commercial License (http:// creativecommons.org/licenses/by-nc/3.0/), which permits unrestricted non-commercial use, distribution, and reproduction in any medium, provided the original work is properly cited. 


\section{Case Report}

A 34-year-old, $162 \mathrm{~cm}, 55 \mathrm{~kg}$ female patient with varicose veins was scheduled to undergo endovenous laser therapy and a varicosectomy. She had no other underlying disorders or medical history of surgery. With the exception of an allergy to dust, she had no other allergic reactions to medications and food, and there was no family history of allergies. There were no abnormalities in the preoperative physical examination, hematological test, simple X-ray test and ECG.

Thirty minutes before arriving in the operation room, 2.0 mg of midazolam and $0.2 \mathrm{mg}$ of glycopyrrolate were injected into the muscle as preanesthetic medication. After arriving in the operation room, the ECG, heart rate, non invasive blood pressure manometer, pulse oximeter, and end tidal $\mathrm{CO}_{2}$ were monitored. The preanesthetic vital signs indicated a blood pressure (BP), pulse rate and oxygen saturation of 110/70 $\mathrm{mmHg}, 75 \mathrm{bpm}$ and 99\%, respectively. To induce general anesthesia, $110 \mathrm{mg}$ of propofol mixed with $40 \mathrm{mg}$ of $2 \%$ lidocaine was injected intravenously. After confirming the patient's loss of consciousness, manual ventilation was performed using a mask with oxygen $3 \mathrm{~L} / \mathrm{min}$, nitro oxide $3 \mathrm{~L} /$ min and, sevoflurane 3 vol\%. Endotracheal intubation was performed one or two minutes after administering $40 \mathrm{mg}$ of rocuronium.

Her heart rate (HR) increased immediately after the endotracheal intubation. Three minutes later, her HR and BP was $135 / \mathrm{min}$ and $75 / 35 \mathrm{mmHg}$, respectively. Suspecting the influence of inhalation anesthetics, the anesthetic medication was discontinued, but the HR continued to increase. At the same time, her systolic and diastolic blood pressure was $60-$ $70 \mathrm{mmHg}$ and $30-35 \mathrm{mmHg}$, respectively. In addition, there was slight flaring observed in the face and chest as well as angioedema around the eyes. Subsequently, the end tidal $\mathrm{CO}_{2}$ showed an obstructive pattern, and urticaria broke out with anaphylaxis. $100 \%$ oxygen was provided promptly at $6 \mathrm{~L} /$ min, with crystalloid and colloid solution. After administering ephedrine $4 \mathrm{mg}$ twice, her vital signs recovered within 10 minutes to indicate a HR and BP of 100/min and $110 / 65 \mathrm{mmHg}$, respectively.

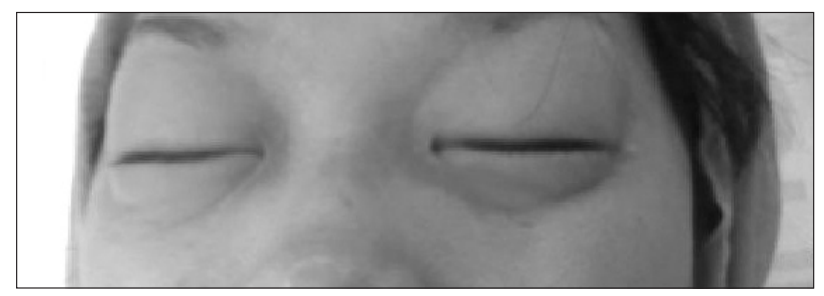

Fig. 1. Patient with facial angioedema.
With time, the whole body flare and urticaria worsened and angioedema was observed around her eyes (Fig. 1). Therefore, $250 \mathrm{mg}$ of hydrocortisone (Solucortef ${ }^{\circledR}$, Pfizer, USA) and 3 mg of an antihistamine, piprinhydrinate (Plokon ${ }^{\circledR}$, Yungjin Pharm. Co., Korea), were injected intravenously followed by $3 \mathrm{mg}$ of midazolam to prevent emergence. Subsequently, her vital signs stabilized about 20 minutes after the induction of anesthesia, the urticaria, flare, and angioedema relived. It was decided that she should be under further observation to determine whether to proceed with surgery. Forty minutes after inducing anesthesia, her vital signs continued to stabilize and the urticaria and angioedema around the swollen eyes were relieved. Therefore, after a discussion with her attending surgeon and parents, a decision was made to proceed with the operation.

Anesthesia was maintained with sevoflurane $2-3 \%$, oxygen and nitro oxide at $2 \mathrm{~L} / \mathrm{min}$. During the two hour operation, no additional muscle relaxant was administered, and the operation was completed without complications. As the operation proceeded, the obstructive pattern of the $\mathrm{CO}_{2}$ curve disappeared and the end tidal $\mathrm{CO}_{2}$ returned to normal. Extubation was performed after postoperative manual ventilation ensured the suitability of the patient's tidal volume and rate, and her vital signs were also within the normal range.

She was transferred to the recovery room after extubation with spontaneous respiration, consciousness, BP, oxygen saturation all normal. The anaphylactic reaction that developed during anesthesia was explained to both the patient and her parents. The risk of side effects during anesthesia due to the pathogenic potential of anaphylaxis to the medication employed was also explained. An intradermal skin test was advised for a differential diagnosis.

The patient wanted to take the test during her hospitalization, and on post operative day 2 , an intradermal skin test was performed at the department of dermatology at our hospital to check the medications administered at the induction of anesthesia as well as the drugs used in subsequent anesthesia. Histamine was taken as a positive control while a saline solution was used as the negative control; $0.03 \mathrm{ml}$ of each test substance was injected on the back of the patient with a hypodermic needle in the form of a clinically used solution diluted at ratio of $1: 10,1: 100$, and $1: 1,000$ from a stock solution. Twenty minutes after the injection, the size of any wheal was measured. The criterion of positivity to an intradermal skin test is the mean diameter of the measured injection papule being larger than the diameter of the wheal induced by the positive control solution or twice than that of the bleb produced by the first injection. The test was stopped every time a positive reaction occurred at a lowest density level. Among the medications used to induce anesthesia on the operation day, rocuronium elicited 
Table 1. The Skin Wheal Size after the Intradermal Injection (mm)

\begin{tabular}{|c|c|c|c|c|}
\hline & $1: 1,000$ & $1: 100$ & $1: 10$ & $1: 1$ \\
\hline Succinylcholine $(20 \mathrm{mg} / \mathrm{ml})$ & $6.5 / 6.5$ & $6 / 6$ & $6 / 6.5$ & $6 / 7.5$ \\
\hline Rocuronium $(10 \mathrm{mg} / \mathrm{ml})$ & $7 / 7$ & $6 / 9.5^{*}$ & & \\
\hline Vecuronium (2 mg/ml) & $5 / 5$ & $5 / 6$ & $6 / 6.5$ & $6 / 9 *$ \\
\hline Propofol $(10 \mathrm{mg} / \mathrm{ml})$ & $5.5 / 5.5$ & $6 / 6$ & $6.5 / 6.5$ & $6 / 6$ \\
\hline Thiopental (25 mg/ml) & $6 / 6$ & $7 / 7$ & $6 / 6$ & $7 / 7$ \\
\hline Lidocaine $(20 \mathrm{mg} / \mathrm{ml})$ & $5.5 / 5.5$ & $6.5 / 6.5$ & $6 / 7$ & $6 / 6$ \\
\hline Fentanyl $(50 \mu \mathrm{g} / \mathrm{ml})$ & $5 / 5$ & $5 / 5$ & $6 / 6$ & $5 / 5.5$ \\
\hline Midazolam (1 mg/ml) & $5 / 5$ & $5 / 5$ & $6 / 6.5$ & $6 / 6$ \\
\hline Positive control & & & & $7 / 9$ \\
\hline Negative control & & & & $6 / 6$ \\
\hline
\end{tabular}

A/B, A: the skin wheal size before the intradermal injection. B: the skin wheal size 20 minutes later. *Positive reaction.

a positive reaction in a $1 / 100$ dilution, and was identified as the causing agent for the anaphylaxis. In addition, another muscle relaxant, vecuronium, showed a positive reaction in an undiluted solution. The tests with the other drugs showed negative reaction (Table 1).

The patient and her parents were informed that she also had a positive reaction to vecuronium, which is commonly used as a nondepolarizing muscle relaxant for inducting operation. The patient was asked to explain her medical history to medical staff at any future procedure, and she was discharged the next day.

\section{Discussion}

Anaphylaxis may be defined as a severe, even deadly, allergic reaction involving the whole body. It is a type 1 hypersensitivity reaction resulting from the release of vasomotor substances, such as histamine, lukotriene, etc., which are activated by mast cells or basophilic cells via the mediators of immunoglobulin $\mathrm{E}$ (IgE). The clinical symptoms of anaphylactic reactions are difficult to differentiate from anaphylactoid reactions not mediated by IgE, and the distinction was reformulated in recent terminology as immunologic anaphylaxis vs. non-immunologic anaphylaxis [2].

The prevalence of anaphylaxis during general anesthesia varies from $1: 4,500$ to $1: 20,000$. The mortality rates for anaphylaxis are $3-6 \%$ and the frequency of severe neurological damage is $2 \%$. The clinical features involve the following: cardiovascular collapse, such as hypotension, tachycardia, or bradycardia; complications of the respiratory system, such as bronchospasm, pulmonary edema, and hypoxia; and skin reactions, such as systemic lupus erythematosus and edema, etc. Such reactions require immediate medical treatment, which includes the correction of hypoxia, inhibition of the release of chemical mediators by removing the causative agents, supplementation of intravascular volume, immediate provision of $100 \%$ oxygen, administration of epinephrine, and early endotracheal intubation against the occurrence of vascular edema, etc. [3].
The episode of anaphylaxis in our patient was identified with the concurrence of facial edema, airway resistance, bronchospasm like a change in the end tidal $\mathrm{CO}_{2}$ curve, and cardiovascular reactions. Rocuronium was presumed to be the causative agent of anaphylaxis because the injection of rocuronium initiated the increase in airway resistance and the sudden decrease in blood pressure.

Considering that it is impossible to make a precise diagnosis of the causative substance of anaphylaxis during surgery, it is advisable to terminate the operation as early as possible and discontinue any medications [4]. In our case, the patient's condition was not critical and after prompt administration of fluid and ephedrine, her vital signs were maintained continuously in the normal range. In addition a steroid effective in a delaying the reaction was injected into the patient, followed by the administration of antihistamine to treat the vascular edema, even though it was reported that the administration of antihistamine is not effective in episode of anaphylaxis [5]. As a result, her condition improved considerably, and the operation was resumed after a discussion with her attending surgeon and parents. She recovered from the operation without complications.

Vascular edema is a skin change resulting from skin vasodilation and an increase in vessel wall permeability. When vascular edema is dilated, the transition of a large amount of fluid takes place, and the mucosal edema in the pharynx, larynx, trachea, etc. causes an obstruction of the upper airway, which affects the entire trachea. An increase in exudate can lead to hypovolemic shock [2]. Vascular edema can be attributed not only to acute allergic reactions but also to hereditary vascular edema due to a $\mathrm{Cl}$ esterase inhibitor deficiency and to acquired vascular edema by drugs, such as an ACE inhibitor, autoimmune diseases, and various underlying disorders such as cancer [6]. Despite the diverse causes, the clinical manifestations, such as airway edema, are so similar that differentiation is required. In the present case, where the patient had no abnormality in her medical history, a C4 blood test, which is one of the screening 
tests for acquired vascular edema, was normal. Therefore, an allergic reaction to the administered drugs was assumed to be the cause.

Anaphylaxis can occur as a result of any medication used during anesthesia, among which $60 \%$ of cases are connected to muscle relaxants, $20 \%$ to latexes, $15 \%$ to antibiotics, and the rest $5 \%$ to sleeping pills, colloids, opioids, etc. Considering that her previous medical history, such as atopy, asthma, allergy to food or dust, etc., can increase the risk of allergy to latex, but not to muscle relaxants, her experience of allergy to dust did not appear to have any relevance to anaphylaxis [7].

The diagnosis of anaphylaxis while its reaction is active, includes the measurement of tryptase, histamine, complement or IgE, skin prick test, intradermal skin test, etc. A skin prick test can be used for a differential test because it is simple, reliable, and can test several antigens simultaneously with relative safety. On the other hand, an intradermal skin test has high sensitivity with a low false-negativity rate, showing much greater sensitivity than a specific IgE measurement. In addition, an intradermal skin test is regarded as a critical criteria of diagnosis for allergic reactions that develop during operation, particularly muscle relaxant-induced allergic reactions $[8,9]$.

In the intradermal skin test performed in our case, rocuronium showed a positive reaction in a 1/100 dilution, while succinylcholine, which known to be relatively more risky, showed a negative reaction. The reason why the patient developed an allergic reaction, even though she had never been exposed to muscle relaxants, is that quaternary ammonium ions are present in common household goods, such as tooth paste, collyrium, shampoo, antiussive (cough medicine), which may have induced sensitization response. The difference in the prevalence of an allergic response to muscle relaxants between several countries varies up to six times. It was reported that such a difference may be related to the frequency of using these daily goods [10]. The reason why both rocuronium and vecuronium showed positive reactions may be due to the following possibilities: first, cross-sensitization response by quaternary ammonium ions that muscle relaxants commonly share second, false positive reaction to substances other than the antigens, caused by a hyper-response developed because the intradermal skin test in our case was conducted much earlier than the generally recommended waiting period of 4 weeks after the onset of symptom [11]. It was reported that the rate of false positive reactions increases when an undiluted or a low dilution ratio solution is used in the intradermal skin test and that a skin prick test on healthy adults with undiluted rocuronium and vecuronium showed $50 \%$ and $40 \%$ false positive reaction, respectively [12]. In addition, a recent study suggested that a positive reaction only at more than $1 / 100$ dilution should be regarded as meaningful [13]. A review of the recent literature showed that rocuronium may have been the cause of anaphylaxis for our patient. However, the possibility that the positive reaction to vecuronium is a false positive cannot be ruled out.

An anaphylactic reaction to rocuronium was reported to increase with increasing frequency of its use, and a crossreaction may developed as a result of the quaternary ammonium ions as the epitope of the muscle relaxant. Therefore, when a patient shows an allergic reaction to a muscle relaxant, it is necessary to confirm thecross reaction to other muscle relaxants [14,15].

Providing the proper treatment in the event of anaphylaxis is important but pretreatment to prevent exposure to its inducers is much more important. It is practically impossible to perform a skin test on every medication administered during anesthesia for every patient. However, in patients with a potential risk of anaphylaxis, such as patients who have experienced an allergic response to a medication, it is necessary to perform a skin test to selectively identify the inducing materials. When responsible agents are identified, the administration of the causative agents and cross reaction-induced substances should be avoided. If there is no alternative but to employ the inducers, an antihistamine and steroid should be administered as a premedication prior to surgery, and the medication should be diluted and injected slowly or in small doses with a gradual increase to reduce anaphylactic reaction as much as possible.

An anaphylactic reaction during surgery is rare but possible. In addition, the diagnosis of anaphylactic reactions is difficult to make during the induction of anesthesia when various types of medication are used simultaneously. Therefore, it is essential to understand clearly the criteria for a diagnosis and treatment of anaphylaxis, to make a prompt diagnosis, and to provide a proper treatment for its occurrence. In this case, the operation was performed safely after the incidence of anaphylaxis caused by rocuronium, and the responsible agent was identified using the intradermal skin test, which has higher sensitivity than other diagnostic methods.

\section{References}

1. Mertes PM, Laxenaire MC, Lienhart A, Aberer W, Ring J, Pichler WJ, et al. Reducing the risk of anaphylaxis during anaesthesia: guidelines for clinical practice. J Investig Allergol Clin Immunol 2005; 15: 91-101.

2. Joseph T. Nitti: Anesthetic Complications. In: Clinical Anesthesiology. 4th ed. Edited by Morgan Jr, Maged SM, Michael JM: USA, McGraw-Hill. 2006, pp 970-3.

3. Mertes PM, Laxenaire MC, Alla F. Anaphylactic and anaphylactoid reactions occurring during anesthesia in France in 1999-2000. Anesthesiology 2003; 99: 536-45.

4. Lieberman P. Anaphylactic reactions during surgical and medical 
procedures. J Allergy Clin Immunol 2002; 110(2 Suppl): S64-9.

5. Fisher MM, Munro I. Life-threatening anaphylactoid reactions to muscle relaxants. Anesth Analg 1983; 62: 559-64.

6. Jesen NF, Weiler JM. C1 esterase inhibitor deficiency, airway compromise, and anesthesia. Anesth Analg 1998; 87: 480-8.

7. Porri F, Lemiere C, Birnbaum J, Guilloux L, Lanteaume A, Didelot $\mathrm{R}$, et al. Prevalence of muscle relaxant sensitivity in a general population: implications for a preoperative screening. Clin Exp Allergy 1999; 29: 72-5.

8. Dybendal T, Guttormsen AB, Elsayed S, Askeland B, Harboe T, Florvaag E. Screening for mast cell tryptase and serum IgE antibodies in 18 patients with anaphylactic shock during general anesthesia. Acta Anaesthesiol Scand 2003; 47: 1211-8.

9. Hwang SM, Suh JW, Lim SY. Anaphylaxis after Thiopental and Rocuronium Induction. Korean J Anesthesiol 2005; 48: 95-8.

10. Florvaag E, Johansson SG, Oman H, Venemalm L, Degerbeck F, Dybendal T, et al. Prevalence of IgE antibodies to morphine. Relation to the high and low incidences of NMBA anaphylaxis in Norway and Sweden, respectively. Acta Anaesthesiologica Scandinavica 2005; 49: 437-44.

11. Ebo DG, Hagendorens MM, Bridts CH, De Clerck LS, Stevens WJ. Scandinavian clinical practice guidelines on the diagnosis, management and follow-up of anaphylaxis during anaesthesia: some diagnostic issues. Acta Anaesthesiol Scand 2008; 52: 314-5.

12. Dhonneur G, Combes X, Chassard D, Merle JC. Skin sensitivity to rocuronium and vecuronium: a randomized controlled prick testing study in healthy volunteers. Anesth Analg 2004; 98: 986-9.

13. Levy JH. Anaphylactic reactions to neuromuscular blocking drugs: are we making the correct diagnosis? Anesth Analg 2004; 98: 881-2.

14. Matthey P, Wang P, Finegan BA, Donnelly M. Rocuronium anaphylaxis and multiple neuromuscular blocking drug sensitivities. Can J Anaesth 2000; 47: 890-3.

15. Lee HM, Song SO. Ananaphylaxis after Injection of Rocuronium. Korean J Anesthesiol 2006; 51: 101-4. 